\title{
THEORETICAL LINE PROFILES IN THE ULTRAVIOLET SPECTRA OF EARLY B-TYPE STARS
}

\author{
by Donald C. MorToN \\ (Princeton University Observatory, U. S. A.)
}

RÉsumé. - On a calculé les profils de 106 raies $U$. V. de longueurs d'onde comprises entre 911,6 et $3000 \AA$, y compris celles de largeur équivalente supérieure à $2 \AA$ et cela pour des modèles d'atmosphère $B_{\mathrm{z}} V$ et $B_{0} V . L a$ plupart des raies se situent dans la région de longueur d'onde inférieure à $1410 \AA$ : elles présentent une absorption centrale et beaucoup ont des ailes très étendues.

ABstract. - The blended profiles of 106 ultraviolet lines, including all those expected to have equivalent widths greater than $2 \AA$ in the wavelength range 911,6 to $3000 \AA$, have been computed for $B_{z} V$ and $B_{0} V$ model atmospheres. Most of the lines are found shortward of $1410 \AA$, where they have deep corts and many have extensive wings. This blanketing amounts to $31 \%$ at $B_{\mathbf{z}} V$ and $20 \%$ at $B_{0} V$.

Резюме. - Вычислены контуры 106 ультрафиолетовых линий с длиной волн заключенной между 911,6 и $3000 \AA$, включая и те эквивалентная ширина которых превышает $2 \AA$ для атмосферных моделей $\mathrm{B}_{\mathrm{z}} \mathrm{V}$ и $\mathrm{B}_{\mathrm{o}} \mathrm{V}$. Большинство линий расположено в области длины волн меньше $1410 \AA$; они выявляют наличие сильного центрального поглощения и многие имеют очень распростерты крылья.

Rocket-borne instruments soon will provide ultraviolet spectra of hot stars with resolutions of about $1 \AA$. Consequently it is important to attempt to predict these spectra using the best theory presently available and including the profiles of the strongest lines.

As a basis for these calculations, two early-type models by Miss UNDERHILL (1962a) were chosen, both with surface gravities of $10^{4} \mathrm{~cm} \mathrm{~s}^{-2}$ and abiundances by mass of 0.68 hydrogen and 0.32 helium. One had an effective temperature of $25673 \mathrm{oK}$ and a spectral type near B2 V, while the other has a temperature of $31023{ }^{\circ} \mathrm{K}$ and a type near Bo V. Electron scattering and the bound-free absorption of $\mathrm{HI}, \mathrm{H}-, \mathrm{He} \mathrm{I}$, and $\mathrm{He}$ II were included in the opacity, but no line absorption.

106 lines were chosen, primarily from the list of GAUSTAD and SPITZER (1961), and are expected to include all those with equivalent widths greater than $2 \AA$ in these atmospheres. The adopted abundances are given in Table $I$. The broadening in the Lyman lines of hydrogen was represented by a simplified expression derived by GRIEM (1960) which includes the perturbations of ions by the quasi-static approximation and the perturbation of electrons by the impact approximation. For all other lines, a Doppler-damping profile was assumed, with a
TABLE I

Abundances of the elements by weight.

\begin{tabular}{cccc}
\hline \hline Element & Abundance & Element & Abundance \\
- & $\overline{-}$ & $\overline{\mathrm{AI}}$ & $0.45 \times 10^{-4}$ \\
$\mathrm{H}$ & 0.68 & $\mathrm{Si}$ & $8.8 \times 10^{-4}$ \\
$\mathrm{He}$ & 0.29 & $\mathrm{~S}$ & $6.7 \times 10^{-4}$ \\
$\mathrm{C}$ & 0.0067 & $\mathrm{CI}$ & $8.8 \times 10^{-4}$ \\
$\mathrm{~N}$ & 0.0014 & $\mathrm{~A}$ & $6.3 \times 10^{-4}$ \\
$\mathrm{O}$ & 0.015 & $\mathrm{Fe}$ & $2.2 \times 10^{-4}$ \\
& & &
\end{tabular}

damping constant ten times the classical value so that

$$
\delta=\frac{10}{4 \pi} \frac{8 \pi^{2} e^{2}}{3 m_{e} c} \frac{1}{\lambda_{0}^{2}}=\frac{0.177}{\lambda_{0}^{2}} \mathrm{~s}^{-1} .
$$

The effect of the blended profiles on the emergent flux was computed using a programme provided by Miss UNDERHILL (1962b). . A set of 660 wavelengths were chosen from 911.6 to $3000 \AA$, spaced according to the irregularities in the spectrum. At each wavelength the line absorption was summed over all contributing profiles and 
added to the continuous opacity for 44 levels in the atmosphere. The transfer equation was then solved at each wavelength to give the emitted flux, but no attempt was made to iterate the model for flux constancy throughout the atmosphere. All lines were assumed to be formed in pure absorption. The calculation of a few profiles formed by pure coherent scattering showed no major differences from those in absorption. Pussible nonequilibrium processes could affect the cores of the lines but it is unlikely that they could cause strong emission lines as in the Sun because in these stars it would be difficult for such processes to enhance lines above the ultraviolet continuum which carries most of the flux.

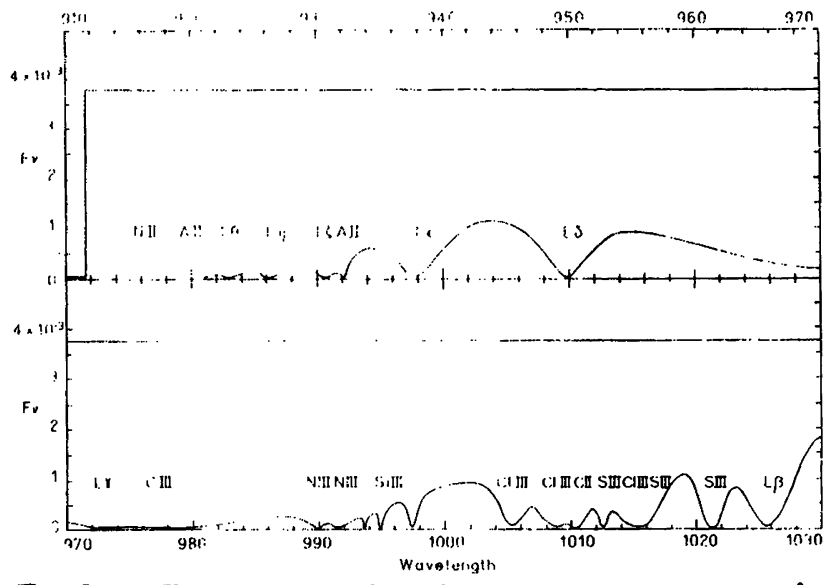

Fia. 1. - The spectrum of a B2V star from 910 to $1030 \AA$.

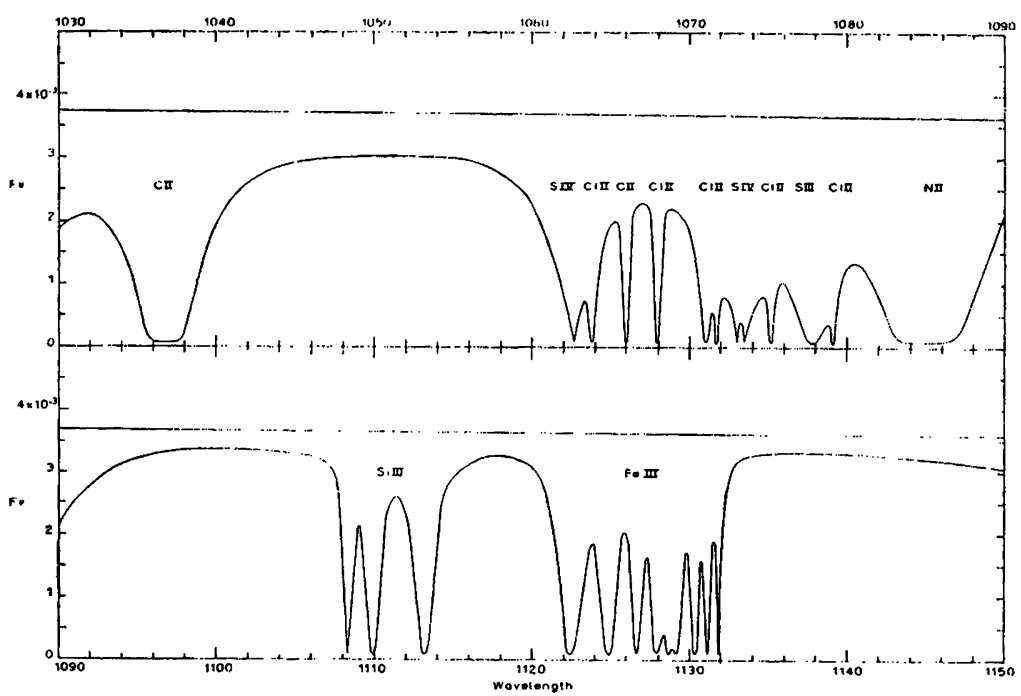

Fra. 2. - 'Tho spectrum of a B2V star from 1030 to $1150 \AA$.

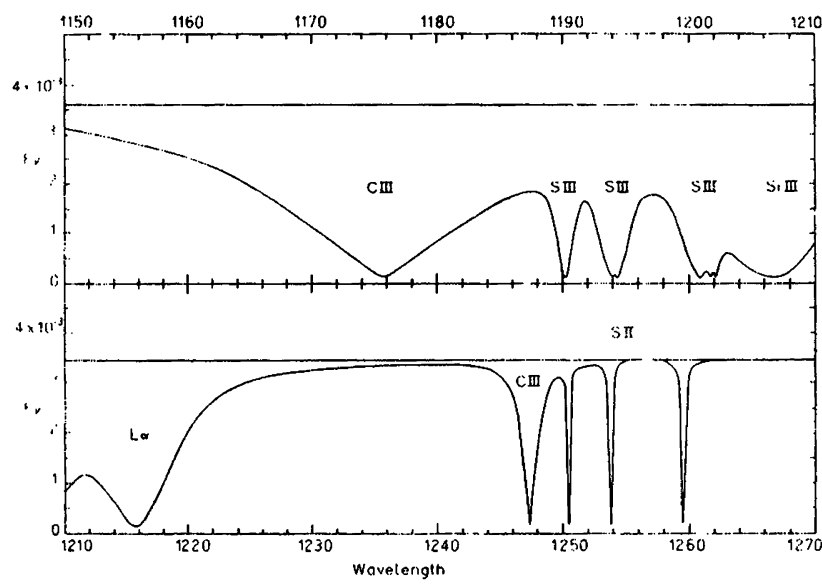

Fra. 3. - The spectrum of a B2V star from 1150 to $1270 \AA$

Figures 1-5 show the calculated spectrum of the B2 V star. The continuum flux is represented by the nearly horizontal lines across the upper parts

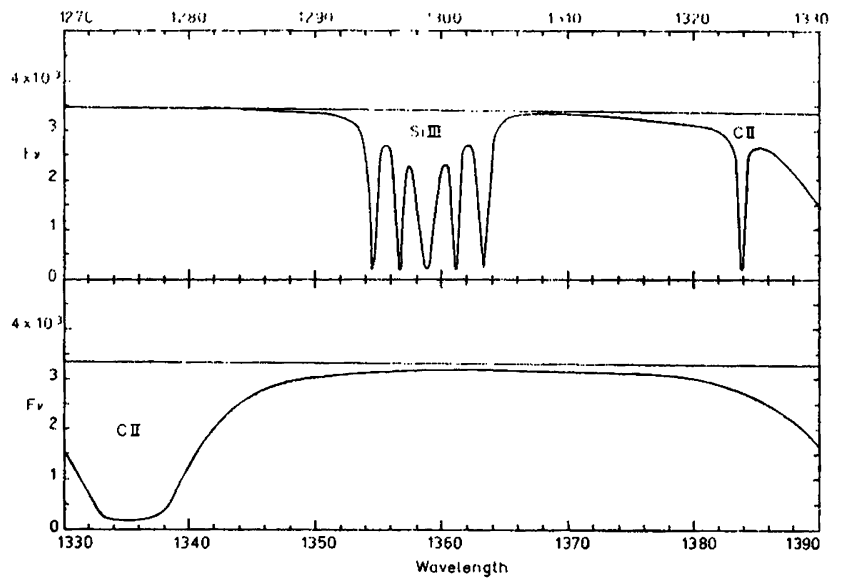

Fic. 4. - The spectrum of a B2V star from 1270 to $1390 \AA$.

of the diagrams. It is seen that many of the lines are rather strong, absorbing a large fraction of the continuum. The cores of all lines are very deep, a 


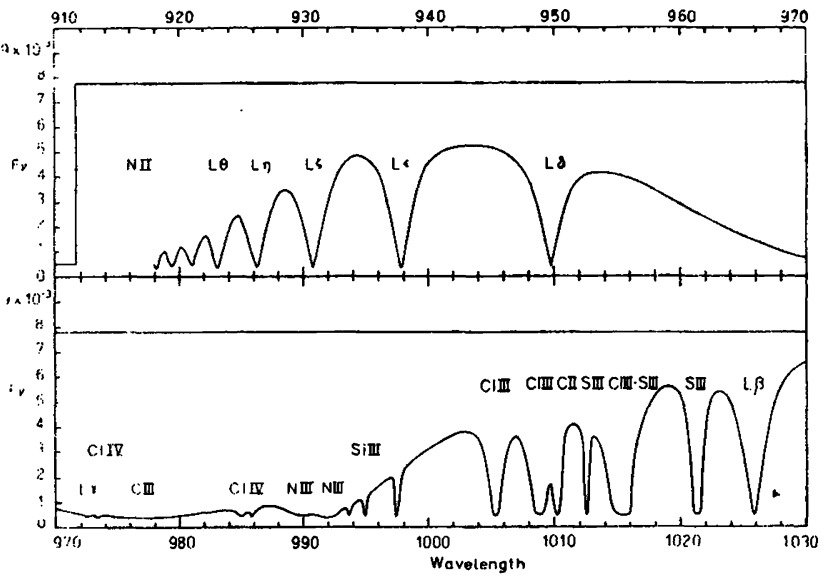

Fia. 5. - The speotrum of a B2V star from 1390 to 3000 A.

consequence of the low temperature in the outermost layers and the low continuous opacity at these wavelengths. However, the precise values of the central intensities are not reliable because at these points the opacity is so high that optical depth unity is reached in one or two integration steps. From 1560 to $3000 \AA$ there are no strong lines. In Figure 6 the first $120 \AA$ of the B0 V spectrum longward of the Lyman limit is reproduced to show the general characteristics. Here the Lyman series and most of the other lines are somewhat weaker so that more of the continuum is visible. A fow lines in the higher ionization

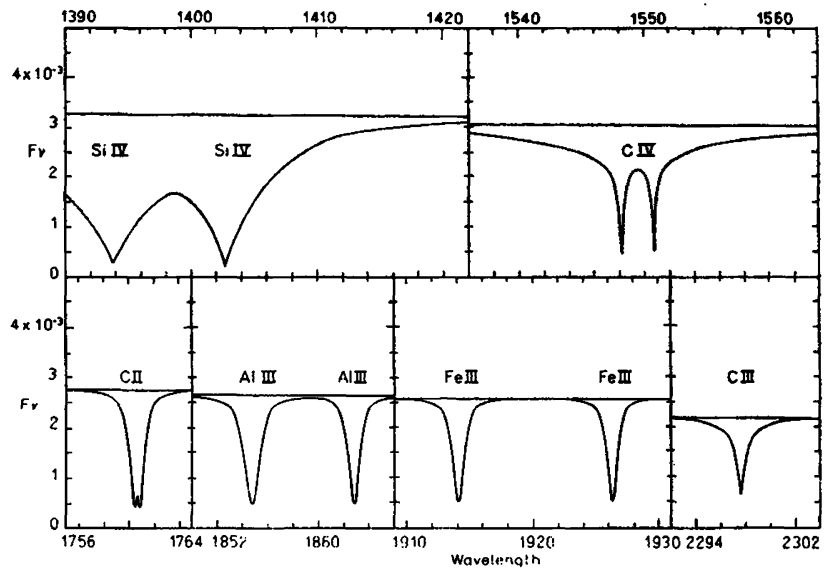

Fre. 6. - The spectrum of a B0V star from 910 to $1030 \AA$.

states are stronger. The He II line at $1640 \AA$, corresponding to $\mathrm{H} \alpha$, is only $1 \AA$ wide. In the B2 V model the ultraviolet blanketing totals $31 \%$, while in the B0 V model it is $20 \%$. These results demonstrate the necessity of including the opacity of the strongest metal lines as well as the Lyman series when calculating early-type model atmospheres. A programme of constructing fluxconstant models with the lines has been begun at Princeton in collaboration with Dimitri MrHauls.

A more complete account of the present work has been submitted to the Astrophysical Journal, 1965, 141, 564.

Manuscrit reçu le 20 aout 1964.

\section{REFERENCES}

Gadstad J. E. and Sptizer L. S., 1961, Ap. J., 134, 771.

GrIEM H. R., 1960, Ap. J., 132, 883.
Undernill A. B., 1962a, Publ. Dom. Astrophys. Obs., 11, 433; 1962b, Publ. Dom. Astrophys. Obs., 11, 467.

\section{Discussion (*)}

A. J. DeUTsor. - Last week, in Utrecht, Dr. WroBaL reported some work by himself and Dr. KRAFT on some colorimetric effects of the gravity darkening associated with stellar rotation in stars somewhat cooler than these. Krapt and WruBre have found some such effects ; they are small but of the same order as those often associated with changes in (H/M). Of course, we know that the $B$ stars described here are also likely to be rapid rotators, in general ; and, therefore, we must be on guard against misinterpretation of the colorimetric effects of gravity darkening.

Y. Onmar. - I wonder if the new calculations describe also the luminosity effects shown by the Balmer continuum, the one discovered by Chalongr and BARBIER for B stars and the one found by IWANowskA and myself for $F$ stars.

S. E. STrom. - The grid of models we have calcu-

(*) This discuseion is relevent to the four preceeding papers. lated have only been computed for two values of $\log g$. Thus a qualitative comment is all that is possible. The Balmer discontinuity is 'decreased, for all $T_{\text {eft }}$ values in the range between 10000 and $20000 \mathrm{KK}$, as $\log g$ decreases from 4,0 to 3,0. For a model having $\mathrm{T}_{\text {eff }}=9500 \mathrm{oK}$ and $\log g$ values of 4,$3 ; 4,0$ and 3,7 , the values of the Balmer discontinuities are respectively $\mathrm{D}=0,55 ; 0,57$ and 0,58 . These models do not include the higher members of the Balmer series and thus the observed and the above given values of $\mathrm{D}$ are not directly comparable. The change in the theoretical value of $\mathrm{D}$, however, is well represented by the above sample set.

M. S. SAVEDOFF. - How does U. V. (2000 $\AA)$ and visual $(4000 \AA)$ opacity compare?

S. E. STrom. - For a model with $T_{\text {eff }}=10^{4} \mathrm{oK}$, the values of the monochromatic optical depths differ by about a factor 2 in the sense that those at $2000 \AA$ 
are larger. For higher $\mathrm{T}_{\text {eff }}$, the values of the monochromatic opacities are again quite comparable. A complete table of monochromatic optical depths at selected wavelengths will appear for each of my models (in the effective temperature range 10000 to $20000 \mathrm{~K}$ ) in a forthcoming Ap. J. Supplement. If the models correctly predict the flux at $4000 \AA$, then, they should be valid for predicting the flux at $2000 \AA$, unless, of course, there is some unknown opacity source. The results presented at this meeting, generally support the view that, at least for stars whose rotational velocities are not large, the models and observations are in fairly good agreement.

L. Gratton. - I would like to ask Miss UNDERHmL whether she thinks that deviations from hydrostatic equilibrium would cause a kind of extended-atmosphere-effect which might cause a still larger discrepancy between observed and computed fluxe.s, making the calculated U. V. fluxes still larger ?

Miss A. B. UNDERHIL. - My model atmosphere computations are in essential agreement with those of AvRETT and STrom as regards the various effects they have discussed. The models all give about the same results for the continuous spectrum. It is when you begin to compare computed line profiles with observed line profiles (not $\mathrm{H}$ lines) in $\mathbf{B}$ stars that the discrepancies appear. It turns out that the lines are formed high in the model where the conditions of radiative and hydrostatic equilibrium may not be enough to define the physical conditions affecting line formation. I and Miss C. Gunludume have also been experimenting with a pseudo representation of line blanketing. We find that for a model in which $T$ near $\tau=.1$ is kept constant, more or less (in order to preserve the line spectrum in the $4000 \AA$ region), the $T_{\text {eff }}$ is reduced by 500 to $1000{ }^{\circ} \mathrm{K}$ and $F_{v}$ at $\lambda<1500 \AA$ is reduced somewhat while $F_{v}$ for $\lambda>2000 \AA$ is increased. A $\Delta$ mag $\lambda 5560 \AA$ of 0,2 or 0,5 may be achieved in comparison to the results without line blanketing at $\lambda<1400 \AA$.
Miss A. B. UNDErHILL. - Spectral types in the beginning represent the observed strengths of strong lines. Such lines are not well predicted by the models; later correlations have been made between colours or Balmer jumps, D and spectral type. The continuous spectrum models predict colours or values of $D$ quite well. I think it better to relate to stars via these parameters directly and not via spectral types. Thus U. V. observations can relate to colours or $\mathrm{D}$ and to models, but do not expect the same $\mathrm{D}$ or colours always to refer to the same spectral type.

S. E. STrom. - I would agree with Miss UNDERH⿰L's comments and would add that near Ao, even for stars having identical $T_{\text {eff }}$ values, the metal opacities due to $\mathrm{Si}$ and $\mathrm{Mg}$ may significantly affect the Balmer discontinuity. Recent abundance analyses of Sirius by S. Strom and K. Strom (unpublished) and BOYARCHUCK indicate that the above metals are overabundant by a factor of 10 to 20 and, if this is so, the Balmer discontinuity will be significantly decreased.

S. E. Strom. - We have calculated for all our models between $10000{ }^{\circ} \mathrm{K}$ and $20000 \mathrm{~K}$ the details of the spectrum of the higher Hydrogen lines. This may be useful in discussing various luminosity-sensitive observed parameters.

Miss A. B. UNDERHILL. - It is interesting to note that in the central parts of the strong lines, you have shown $l_{v} / K_{v}$ may be of the order of $10^{5}$. This means that the central parts of the lines will be formed in the extreme outermost parts of the atmosphere. Models developed using continuous opacity only are not usually defined in adequate detail in these regions ie $\tau_{\text {cont }} \sim 10^{-5}$. Observations of the line centers in the U. V. may give us very interesting information about the physical conditions in the outermost parts of B type atmospheres. The monochromatic optical depths at $2000 \AA$ is about the same as that at $4200 \AA$. It is only in continuum wavelengths at shorter wavelengths that one can hope to observe to deeper layers in the atmosphere than one does in the $4000 \AA$ region. 